Article

\title{
Quantitative Assessment of Trace and Macro Element Compositions of Cassava (Manihot esculenta) Storage Roots Enriched with B-Carotene as Influenced by Genotypes and Growing Locations
}

\author{
Emmanuel Oladeji Alamu ${ }^{1, *}$, Busie Maziya-Dixon ${ }^{2}$ and Alfred Gilbert Dixon ${ }^{2}$ \\ 1 International Institute of Tropical Agriculture, Food and Nutrition Sciences Laboratory, Southern Africa, \\ Research and Administration Hub (SARAH) Campus P.O. Box 310142, Chelstone, Lusaka 10101, Zambia \\ 2 International Institute of Tropical Agriculture, Food and Nutrition Sciences Laboratory, PMB 5230 Ibadan, \\ Oyo State, Nigeria; b.maziya-dixon@cgiar.org (B.M.-D.); a.dixon@cgiar.org (A.G.D.) \\ * Correspondence: o.alamu@cgiar.org
}

Received: 27 October 2020; Accepted: 2 December 2020; Published: 8 December 2020

\begin{abstract}
Cassava's important mineral contents depends on some factors, including genetic and growing locational factors. The study aimed to evaluate the influence of genotype and growing locations on the mineral concentrations in yellow-fleshed cassava root genotypes. Twenty-five pipeline yellow-fleshed cassava genotypes and three white-fleshed varieties (check samples) were planted at five different experimental fields for two seasons, each representing the major agroecological zones in Nigeria. Standard laboratory protocols were employed in the sampling to ensure zero contamination, and the trace and macro elements were determined using the inductively coupled plasma optical emission spectroscopic method (ICPOES). The trace and macro elements identified in all the genotypes and varieties investigated were $\mathrm{Fe}, \mathrm{Mn}, \mathrm{B}, \mathrm{Cu}, \mathrm{Mo}, \mathrm{Co}, \mathrm{Ni}, \mathrm{Zn}$, and $\mathrm{Al} ; \mathrm{Ca}, \mathrm{Mg}, \mathrm{Na}$, K. P, and $\mathrm{S}$ respectively. Genotype and growing location had a highly significant $(p<0.05)$ effect on all the trace elements except $\mathrm{Ti}$ and $\mathrm{Cr}$. However, there was no interactive effect between genotype and growing location on all the trace elements except for $\mathrm{Pb}$ and $\mathrm{Zn}$. Among the explanatory variables, the variable growing location was the most influential on macro and trace elements. Conclusively, genotypes 01/1442 and 01/1273 have outstanding trace and macro element concentrations.
\end{abstract}

Keywords: yellow-fleshed cassava; macronutrient; micronutrient; genotypic variations; growing locations

\section{Introduction}

Cassava has primarily been identified as one of the staple foods that is low in nutritional quality, and this due to the low protein content of its storage roots [1,2]. However, cassava contains $\mathrm{Fe}, \mathrm{Cu}$, $\mathrm{Ca}, \mathrm{Zn}, \mathrm{Mg}$, and $\mathrm{K}$, but most of these minerals are found more in the root peel than in the peeled root, a result of processing [3]. This makes the mineral content in cassava lower than sorghum and maize but comparable with many legumes except soybean [2]. Thus, cassava nutritional value can be considered lower than cereals, legumes, and some other roots and tubers. The importance of cassava as food in sub-Saharan Africa (SSA), especially Nigeria, has proved beyond doubt that it is an economic crop. Besides, it is an easily cultivated subsistence crop, a drought-tolerant, multiple-year crop that can be kept in the ground until needed; this qualifies cassava as a food security crop. Nutritionally, it provides an economical source of dietary energy in the form of carbohydrates [4]. The usefulness of cassava does not end with the roots; its leaves also are used in the preparation of relishes whose protein quality compares favorably with soybean with high levels of vitamins and minerals. 
Hidden hunger, also known as micronutrient deficiencies, happens when the vitamin and mineral intake or absorption is very low to keep the good health and development in children and everyday physical and mental function in adults [5]. It is as a result of the unbalanced nature of diets consumed by people in most of the developing countries, especially Nigeria. Micronutrients deficiency is a challenge faced by the entire world. In most developing countries, deficiencies of $\mathrm{Fe}$ and $\mathrm{Zn}$ are part of the five micronutrients malnutrition that have been identified to be of public health significance. Biofortification is one of the approaches employed by plant breeders to increase the micronutrient content of crops using conventional breeding, such as cassava. There are different types of cassava varieties based on their peel and pulp colors. Generally, the peel appears cream, brown, light brown, and pinkish brown. At the same time, the pulp's appearance could be yellow, orange, cream, and white. The color usually indicates the beta-carotene and moisture content [6]. The white pulp has been the most cultivated in West Africa and especially, Nigeria. The yellow root has its origin traced to the Amazon basin, which over the years, has been cross-bred with the African white varieties using conventional breeding techniques to boost the carotenoid and micronutrient concentrations in cassava [7].

Minerals are essential elements that are not synthesized by the body but are essential for the body to function optimally [8]. These can be grouped into trace and macroelements. They function in overlap with other macronutrients and vitamins to ward off sicknesses and, most importantly, degenerative diseases. These minerals are actively involved in the development of biochemical processes, in which their essential roles are catalytic, functional, and structural [9]. These can be divided into trace and macro elements. Trace or microelements are usually needed in very minute quantities, while macro elements are needed in large quantities in the body [9]. Macro and trace elements have very varied functions, e.g., as electrolytes, as enzyme constituents, and as building materials, e.g., in bones and teeth. Microelements include $\mathrm{Fe}, \mathrm{Zn}, \mathrm{Mn}, \mathrm{B}, \mathrm{Cu}, \mathrm{Ni}, \mathrm{Al}, \mathrm{Ti}, \mathrm{Cr}$, and $\mathrm{Pb}$. Macro elements include $\mathrm{Ca}, \mathrm{Na}$, $\mathrm{K}, \mathrm{P}$, and $\mathrm{S}$.

However, it is of importance to note that the concentration of these micronutrients contained in plant organs depends on several interacting factors, including soil type, plant type/variety, and the growing locations and its management $[2,10]$. As with all crops, the nutritional composition of roots and tubers varies from place to place depending on the climate, soil, crop variety, and other factors [11]. The mineral content of cassava, as stated earlier, depends on several factors, including genetic and growing locational factors. It could be inferred that there is a direct relationship between mineral concentrations in cassava roots and the elemental concentration present in the soil [12]. Mohan et al. [13] studied the mineral composition of nineteen cassava varieties. They discovered that $\mathrm{K}$ is the most abundant mineral present in cassava tubers with values ranging from $3270-10,870 \mathrm{mg} / \mathrm{kg}$. In contrast, the second most abundant mineral is Ca in the range of $1083-1858 \mathrm{mg} / \mathrm{kg}$ the average Ca content reported was $1502 \mathrm{mg} / \mathrm{kg}$, and $\mathrm{Mg}$ in cassava tuber showed a range of $540-895 \mathrm{mg} / \mathrm{kg}$ with a mean content of $740 \mathrm{mg} / \mathrm{kg}$. The S content ranged from $1000-1290 \mathrm{mg} / \mathrm{kg}$ while Fe content varied from $68-248 \mathrm{mg} / \mathrm{kg}$, Mn content ranged from $15.40-33.2 \mathrm{mg} / \mathrm{kg}$. Zn content ranged from $15.0-28.2 \mathrm{mg} / \mathrm{kg}$, $\mathrm{Cu}$ content ranged from $2.2-4.2 \mathrm{mg} / \mathrm{kg}$, and B content ranged from $4.12-7.62 \mathrm{mg} / \mathrm{kg}$. Efforts of several researchers to increase the mineral content of cassava tubers have assisted in improving the nutritional load of some varieties [14,15]. Biofortified crops, such as yellow-fleshed cassava roots could provide an excellent source of certain micronutrients and macronutrients for people that could not be reached by other interventions.

The research aimed to evaluate the impact of genotype and growing locations on the macro and trace elements in yellow-fleshed cassava root genotypes. Cassava breeders, processors, and dieticians will use the research findings from this study in identifying which cassava genotypes is suitable for cultivation in all agro-ecologies and contain optimum nutrients capable of combating hidden hunger. 


\section{Materials and Methods}

\subsection{Fresh Cassava Root Genotypes}

A total of 25 yellow-fleshed cassava genotypes from unlimited yield trial (UYT) and three white roots varieties used as checks (30,572, TME 1, and 91/02324), were collected from the multi-growing locations field trial at five different research farms of the International Institute of Tropical Agriculture, Ibadan, Nigeria. The five trial growing locations (Ibadan, Ubiaja, Onne, Mokwa, and Zaria) were from different agroecological zones of Nigeria (Table 1). The trial was planted in a complete block design with four replicates and during the rainy season in July. There was no application of fertilizers or herbicides during the experiment. The weeding was done manually when necessary. The roots of all the yellow-fleshed genotypes and white-fleshed varieties were harvested at 12 months after planting (MAP). The two middle rows were harvested per plot, and only two replications were processed for analysis.

Table 1. Characteristics of the five trial growing locations

\begin{tabular}{|c|c|c|c|c|c|c|}
\hline Location & GPS & $\begin{array}{l}\text { Agroecological } \\
\text { Zone }\end{array}$ & Elevation & $\begin{array}{l}\text { Annual } \\
\text { Average } \\
\text { Rainfall }\end{array}$ & $\begin{array}{c}\text { Annual } \\
\text { Average } \\
\text { Temperatures }\end{array}$ & Soil Type \\
\hline Ibadan & $\begin{array}{l}7^{\circ} 38^{\prime} \mathrm{N} \\
3^{\circ} 89^{\prime} \mathrm{E}\end{array}$ & $\begin{array}{l}\text { Forest-savanna } \\
\text { transition }\end{array}$ & 227 masl & $1312 \mathrm{~mm}$ & $20.3-33.8^{\circ} \mathrm{C}$ & $\begin{array}{c}\text { Ferric } \\
\text { Luvisols }\end{array}$ \\
\hline Onne & $\begin{array}{l}4^{\circ} 41^{\prime} \mathrm{N} \\
7^{\circ} 09^{\prime} \mathrm{E}\end{array}$ & Humid forest & 44 masl & $4420 \mathrm{~mm}$ & $22.3-33.4^{\circ} \mathrm{C}$ & $\begin{array}{c}\text { Dystric } \\
\text { Nitosols }\end{array}$ \\
\hline Ubiaja & $\begin{array}{l}6^{\circ} 65^{\prime} \mathrm{N} \\
6^{\circ} 38^{\prime} \mathrm{E}\end{array}$ & Subhumid forest & 287 masl & $1186 \mathrm{~mm}$ & $19.9-32.6^{\circ} \mathrm{C}$ & $\begin{array}{l}\text { Dystric } \\
\text { Nitosols }\end{array}$ \\
\hline Mokwa & $\begin{array}{l}9^{\circ} 28^{\prime} \mathrm{N} \\
5^{\circ} 05^{\prime} \mathrm{E}\end{array}$ & $\begin{array}{c}\text { Southern Guinea } \\
\text { savanna }\end{array}$ & 132 masl & $1149 \mathrm{~mm}$ & $18.1-37.3^{\circ} \mathrm{C}$ & $\begin{array}{l}\text { Dystric } \\
\text { Nitosols }\end{array}$ \\
\hline Zaria & $\begin{array}{l}11^{\circ} 16^{\prime} \mathrm{N} \\
7^{\circ} 63^{\prime} \mathrm{E}\end{array}$ & $\begin{array}{c}\text { Northern Guinea } \\
\text { savanna }\end{array}$ & 687 masl & $1076 \mathrm{~mm}$ & $13.9-35.5^{\circ} \mathrm{C}$ & $\begin{array}{c}\text { Ferric } \\
\text { Luvisols }\end{array}$ \\
\hline
\end{tabular}

\subsection{Sampling Method}

The sampling and sample preparation of the cassava roots for mineral analysis was done using the adapted method described by [16]. There was a selection of three storage roots (large-900-2300 g, medium-500-899 g, and small-200-499 g) and washed thoroughly with potable water to remove dirt and adhered sand particles before air-dried on a clean concrete floor [15]. The peeling of the storage roots was done manually using a stainless-steel knife, and the peeled roots were rinsed with deionized water to remove any contaminants. After peeling, the roots were cut longitudinally (from the proximal end to the distal end) into four equal parts. Two opposite sections from each root of each genotype were taken, combined, manually chopped into small pieces, and mixed thoroughly. A batch of samples from this lot was chosen for the mineral profiling analysis.

\subsection{Determination of Trace Macroelement Content}

The processed samples were placed in a petri dish and dried in an uncorroded conventional oven at $40{ }^{\circ} \mathrm{C}$ for 3 days. After drying, the samples were packed in labelled, mineral-free paper envelopes. The trace and the macro element content were determined using Inductively Coupled Optical Emission Spectrometry (ICP-OES) according to the validated method described by [17] and [15]. About $0.30 \mathrm{~g}$ of the dried sample was weighed to into $50 \mathrm{~mL}$ screw-cap polypropylene tubes and added $2.0 \mathrm{~mL} \mathrm{HNO}_{3}$ and $0.5 \mathrm{~mL} \mathrm{H}_{2} \mathrm{O}_{2}$ to initiate the sample digestion. The samples were made to a final volume of $25 \mathrm{~mL}$ with $18 \mathrm{M} \Omega . \mathrm{cm}$ water before injection. The sample flow rate was $2.0 \mathrm{~mL} \mathrm{~min}^{-1}$, and the total analysis time per sample is approximately $2.5 \mathrm{~min}$. Each sample was run in duplicate. Al concentrations of more than $5-10 \mathrm{mg} / \mathrm{kg}$ are frequently associated with contaminant $\mathrm{Fe}$. Thus, the concentration of $\mathrm{Al}$ of less than $10 \mathrm{mg} / \mathrm{kg}$ was used to determine the quality of the data, and all samples had an Al level of 
less than $10 \mathrm{mg} / \mathrm{kg}$. The trace elements identified in all the genotypes and varieties investigated were $\mathrm{Fe}, \mathrm{Mn}, \mathrm{B}, \mathrm{Cu}, \mathrm{Mo}, \mathrm{Co}, \mathrm{Ni}, \mathrm{Zn}$, and $\mathrm{Al}$, respectively. Besides, the macro elements identified in all the genotypes and varieties investigated were $\mathrm{Ca}, \mathrm{Mg}, \mathrm{Na}, \mathrm{K}, \mathrm{P}$, and $\mathrm{S}$, respectively.

\subsection{Statistical Analysis}

The data generated in this study were subjected to analysis of variance (ANOVA) using Linear Regression Model to establish the relationship between the independent variables and the dependent variables and descriptive statistics using the Statistical Analysis System Software (Cary, NC, USA) [18]. Means were separated using Fisher's protected least significance difference test at $p<0.05$. Principal component analysis (PCA) and hierarchical cluster analysis (HCA) were also utilized.

\section{Results and Discussion}

\subsection{Effect of Genotype and Growing Locations on the Trace Elements of Yellow-Fleshed Cassava Roots}

Goodness-of-Fit Statistics for Minerals across the Five Growing Locations

The linear regression model used for the analysis of variance had a regression coefficient $\left(R^{2}\right)$ for all the dependent variables (genotype and growing locations) that ranged from 0.5-0.9. It implies that $50-90 \%$ of the variability of the independent variables (trace elements) were explained by the three explanatory variables (genotype, growing locations, and two-way interaction between the genotype and growing locations). The mean square error (MSE) ranged from 0.1-30.7, and Mn had the highest while $\mathrm{Cr}$ had the lowest. The lower the MSE, the better the model in predicting the variability of the trace elements. The macro elements in the samples had a regression coefficient $\left(R^{2}\right)$ for all dependent variables ranging from $0.2-0.7$, implying that $20-70 \%$ variability of the independent variables for macro elements were explained by the three explanatory variables as described earlier. Five macroelements were recorded for the yellow-fleshed cassava genotypes studied. The mean square error (MSE) ranged from $4.1 \times 10^{3}-1.47 \times 10^{7}$, with the lowest for $\mathrm{S}$ and the highest for $\mathrm{K}$ The high values for the MSE suggest the model may not be good enough in predicting the variability of macroelements.

Table 2 shows the mean square (MS) of the analysis of variance for the trace and macro elements of yellow-fleshed cassava roots from five growing locations. Genotype and growing locations had a highly significant $(p<0.001)$ effect on all the trace elements except Ti and Cr. However, there was no significant $(p>0.05)$ difference of genotype $\times$ growing locations interaction on all the trace elements, but a highly significant $(p<0.001)$ effect was observed for $\mathrm{Pb}$ and a slight significance $(p<0.01)$ for $\mathrm{Zn}$. It implies that the growing locations and genotype affect the trace element contents of the yellow-fleshed cassava roots. Among the explanatory variables, the variable growing locations is the most influential for all the trace elements except $\mathrm{B}$ and Ti, where genotype is the most influential variable. Thus, the growing locations had a strong effect on the concentration of $\mathrm{Fe}, \mathrm{Zn}, \mathrm{Mn}, \mathrm{Cu}$, $\mathrm{Ni}, \mathrm{Al}, \mathrm{B}$, and $\mathrm{Pb}$, while genotype had a strong influence on $\mathrm{B}$ and Ti concentrations. This can be related to the type of soil used for planting. Acidic soils have been reported to be high in $\mathrm{Al}$ and $\mathrm{Mn}$ concentrations $[19,20]$. Genotype $\times$ growing locations interaction was highly significant for both Fe and Zn among 21 sweet potato genotypes from five growing locations in the eastern region of China [21]. Furthermore, another group of researchers evaluated genotype $\times$ growing locations interaction in recombinant inbred lines of wheat bread grains for Fe and $\mathrm{Zn}$ concentration. Genotype $\times$ growing locations interaction ( $\mathrm{G} \times \mathrm{E}$ ) for $\mathrm{Fe}$ and $\mathrm{Zn}$ accounted for $29.46 \%$ and $23.24 \%$ of the total sum of squares, respectively, and the magnitude of $\mathrm{G} \times \mathrm{E}$ interaction was relatively high [22] 
Table 2. Mean squares (MS) of analysis of variance (ANOVA) for trace and macroelements of yellow-fleshed cassava roots across five growing locations

\begin{tabular}{|c|c|c|c|c|c|c|c|c|c|c|c|}
\hline \multirow{3}{*}{ Source } & & \multicolumn{10}{|c|}{ Parameters } \\
\hline & & $\mathrm{Fe}$ & $\mathrm{Zn}$ & Mn & B & $\mathrm{Cu}$ & $\mathrm{Ni}$ & Al & $\mathrm{Ti}$ & $\mathrm{Cr}$ & $\mathbf{P b}$ \\
\hline & DF & MS & MS & MS & MS & MS & MS & MS & MS & MS & MS \\
\hline Genotype & 27 & $60 * * *$ & $39.7^{* * *}$ & $146.5^{* * *}$ & $2.3^{* * *}$ & $4.9^{* * * *}$ & $0.7^{* * *}$ & $30.1 * *$ & 0.4 & 0.1 & 0.8 \\
\hline Locations & 4 & $285.4^{* * *}$ & $536.3^{* * *}$ & $1773.8^{* * *}$ & 1.33 * & $54.7^{* * *}$ & $7.9^{* * *}$ & 85.2 ** & 0.1 & 0.2 & $13.4^{* * *}$ \\
\hline Genotype & & & & & & & & & & & \\
\hline $\begin{array}{c}\times \\
\text { Location }\end{array}$ & 107 & 24.3 & $11.7^{*}$ & 37.2 & 0.5 & $1.0 * * *$ & 0.3 & 14.0 & 0.3 & 0.2 & $0.8^{* * *}$ \\
\hline Source & & $\mathrm{Ca}$ & $\mathrm{Mg}$ & $\mathrm{Na}$ & $\mathbf{K}$ & $P$ & $S$ & & & & \\
\hline & DF & MS & MS & MS & MS & MS & MS & & & & \\
\hline Genotype & 27 & $\underset{* * *}{2,378,351}$ & $\underset{* * *}{3,054,187}$ & $133,832 * *$ & $\begin{array}{c}63,007,980 \\
* * *\end{array}$ & $\underset{* * *}{153,215}$ & $\begin{array}{c}23,015 \\
* * *\end{array}$ & & & & \\
\hline Locations & 4 & $\underset{* * *}{22,331,768}$ & 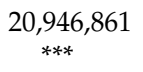 & $\begin{array}{c}589,581 \\
* * *\end{array}$ & $\underset{* * *}{225,500,5890}$ & $\begin{array}{c}9,228,146 \\
* * *\end{array}$ & $\underset{* * *}{200,022}$ & & & & \\
\hline $\begin{array}{c}\text { Genotype } \\
\times \\
\text { Location }\end{array}$ & 107 & 633,863 & $\begin{array}{c}896,698 \\
* * *\end{array}$ & $88,495^{*}$ & $\begin{array}{c}19,347,235 \\
* *\end{array}$ & 81,564 * & $\begin{array}{c}6228 \\
* * *\end{array}$ & & & & \\
\hline
\end{tabular}

DF-Degree of Freedom, ${ }^{* * *},{ }^{* * *},{ }^{*}$ Significant at $p \leq 0.001, p \leq 0.01$ and $p \leq 0.05$ respectively; ns—not significant $p>0.05$.

The growing locations had highly significant $(p<0.001)$ effects on all macroelements. In comparison, genotype had a highly significant $(p<0.001)$ effect on $\mathrm{Ca}, \mathrm{K}$, and $\mathrm{S}$, a significant effect $(p<0.05)$ on $p$, and a slightly significant effect on Na. Genotype and growing locations bring significant $(p<0.05)$ information to explain the variability of the dependent variable $\mathrm{Ca}, \mathrm{Mg}, \mathrm{Na}, \mathrm{K}, \mathrm{P}$, and $\mathrm{S}$. However, among the factors, the growing location is the most influential for the macroelements. Ref. [23] reported that cassava thrives best on soils with adequate macronutrients—especially $\mathrm{Ca}, \mathrm{P}$, and $\mathrm{K}$-coupled with the ability to extract high concentrations of nutrients from the soil. $\mathrm{K}$ plays an essential role in the transport of starch and sugar from the above-ground parts of the plant and, thus, their accumulation in tubers, the reason why it is the macroelement with the highest concentration across all locations and genotypes [24]. The leaves dropping from cassava plants as maturity progresses usually act as mulch for the plant, and traditional farm practices like mulching increases soil $\mathrm{P}$ and $\mathrm{K}$, which in turn prevents a decline in soil $\mathrm{Ca}$ and $\mathrm{Mg}[19,25]$. However, not all soil types have these macro elements in required quantities to support cassava growth because there seems to exist a direct relationship between the concentration of macroelements in cassava roots and their abundance in the soil [24]. Ultimately, a plant's ability to obtain adequate amounts of macronutrients depends critically on the availability of these minerals in the soil concerning factors such as precipitation, temperature, wind, soil type, and soil $\mathrm{pH}[26]$.

\subsection{Trace and Macroelement Content of Yellow-Fleshed Cassava Roots by Genotype}

Tables 3 and 4 show the trace and macroelement content of yellow-fleshed cassava roots by genotype across the five growing locations. The concentrations of $\mathrm{Fe}, \mathrm{Zn}$, and $\mathrm{Mn}$ were found to be higher than $\mathrm{B}, \mathrm{Cu}, \mathrm{Ni}, \mathrm{Al}, \mathrm{Ti}, \mathrm{Cr}$, and $\mathrm{Pb}$, and implies that cassava roots could be a good source of $\mathrm{Fe}, \mathrm{Zn}$, and $\mathrm{Mn}$ which are highly essential micronutrients. The Fe content ranged from $2.6-4.9 \mathrm{mg} / \mathrm{kg}$ (mean $10.4 \pm 5.5 \mathrm{mg} / \mathrm{kg}$ ), Zn from 3.4-30.9 mg/kg (mean $11.1 \pm 4.4 \mathrm{mg} / \mathrm{kg}$ ), and Mn from $1.6-57.8 \mathrm{mg} / \mathrm{kg}$ (mean $14.7 \pm 8.3 \mathrm{mg} / \mathrm{kg}$ ). Also, B had a mean value of $2.0 \pm 0.8 \mathrm{mg} / \mathrm{kg}$, $\mathrm{Cu} 3.2 \pm 1.4 \mathrm{mg} / \mathrm{kg}$, Ni $1.3 \pm 0.6 \mathrm{mg} / \mathrm{kg}$, A1 $5.8 \pm 4.1 \mathrm{mg} / \mathrm{kg}$, Ti $0.5 \pm 0.5 \mathrm{mg} / \mathrm{kg}, \mathrm{Cr} 0.4 \pm 0.3 \mathrm{mg} / \mathrm{kg}$, and $\mathrm{Pb} 1.3 \pm 0.9 \mathrm{mg} / \mathrm{kg}$. On the other hand, among the macroelements, $\mathrm{K}$ has the highest concentrations ranging from $2100-43,000 \mathrm{mg} / \mathrm{kg}$ (mean 10,637 \pm 7172), Ca from 410-7600 mg/kg (mean $1604 \pm 1057$ ), $\mathrm{Mg}$ from 4600-8100 mg/kg (mean $1576 \pm 1077$ ), P from 450-3100 mg/kg (mean $967 \pm 458$ ), S, the lowest, from 138-700 mg/kg (mean $297 \pm 94$ ), while the element with the widest concentration range is $\mathrm{Na}, 4-4100 \mathrm{mg} / \mathrm{kg}$ (mean $167 \pm 297$ ). Chavez et al. [27] reported the average concentrations of five 
trace elements (Fe, Zn, Mn, B, Cu) and six macroelements (Ca, Mg, Na, P, K, S) for 20 yellow-fleshed cassava genotypes. For Fe, the average concentration was $9.6 \mathrm{mg} / \mathrm{kg}$, Zn $6.4 \mathrm{mg} / \mathrm{kg}$, Mn $1.2 \mathrm{mg} / \mathrm{kg}$, B $2.4 \mathrm{mg} / \mathrm{kg}$, and $\mathrm{Cu} 2.2 \mathrm{mg} / \mathrm{kg}$, which are low compared with values in this study except for boron. The concentration reported for the selected macroelements are Ca $590 \mathrm{mg} / \mathrm{kg}, \mathrm{Mg} 1153 \mathrm{mg} / \mathrm{kg}$, $\mathrm{Na} 66.4 \mathrm{mg} / \mathrm{kg}$, P $1284 \mathrm{mg} / \mathrm{kg}$, K $8903 \mathrm{mg} / \mathrm{kg}$, and S $273 \mathrm{mg} / \mathrm{kg}$. All the values fall within the range, as analyzed in this study.

Table 3. Mean value of trace elements of yellow-fleshed cassava roots by genotypes and across five growing locations

\begin{tabular}{|c|c|c|c|c|c|c|c|c|c|c|}
\hline \multirow{2}{*}{ Genotype } & \multicolumn{10}{|c|}{ Parameters (mg/kg) } \\
\hline & $\mathrm{Fe}$ & $\mathrm{Zn}$ & Mn & B & $\mathrm{Cu}$ & $\mathrm{Ni}$ & Al & $\mathrm{Ti}$ & $\mathrm{Cr}$ & $\mathrm{Pb}$ \\
\hline $01 / 1442$ & $14.9 \mathrm{ab}$ & $15.7 \mathrm{ab}$ & $25.8 \mathrm{a}$ & $3.6 \mathrm{a}$ & $4.7 \mathrm{ab}$ & $1.5 \mathrm{abc}$ & $9.4 \mathrm{a}$ & $1.1 \mathrm{a}$ & $0.4 \mathrm{a}$ & $1.6 \mathrm{ab}$ \\
\hline $01 / 1413$ & $14.7 \mathrm{ab}$ & $12.4 \mathrm{abcd}$ & $17.5 \mathrm{abc}$ & $2.7 \mathrm{abcd}$ & $3.4 \mathrm{bcdef}$ & $1.8 \mathrm{ab}$ & $8.6 \mathrm{a}$ & $0.6 \mathrm{a}$ & $0.7 \mathrm{a}$ & $1.6 \mathrm{ab}$ \\
\hline $01 / 1663$ & $13.9 \mathrm{ab}$ & $12.5 \mathrm{abcd}$ & $17.2 \mathrm{abc}$ & $2.6 \mathrm{abcd}$ & 3.1 cdefg & $1.2 \mathrm{abc}$ & $7.9 \mathrm{a}$ & $0.9 \mathrm{a}$ & $0.5 \mathrm{a}$ & $2.3 \mathrm{a}$ \\
\hline $01 / 1115$ & $15.6 \mathrm{a}$ & $15.9 \mathrm{a}$ & $17.0 \mathrm{abc}$ & 2.4 bcde & 4.0 abcd & $1.9 \mathrm{a}$ & $5.8 \mathrm{a}$ & $0.5 \mathrm{a}$ & $0.4 \mathrm{a}$ & $1.4 \mathrm{ab}$ \\
\hline $01 / 1273$ & $12.5 \mathrm{ab}$ & $13.7 \mathrm{abc}$ & $16.4 \mathrm{abc}$ & $2.8 \mathrm{abc}$ & $4.4 \mathrm{abc}$ & $1.3 \mathrm{abc}$ & $9.0 \mathrm{a}$ & $0.7 \mathrm{a}$ & $0.3 \mathrm{a}$ & $1.6 \mathrm{ab}$ \\
\hline $01 / 1335$ & $11.1 \mathrm{ab}$ & $12.6 \mathrm{abcd}$ & $21.5 \mathrm{ab}$ & $2.6 \mathrm{abcd}$ & 3.4 bcdef & $1.5 \mathrm{abc}$ & $8.4 \mathrm{a}$ & $0.7 \mathrm{a}$ & $0.4 \mathrm{a}$ & $1.2 \mathrm{ab}$ \\
\hline $94 / 0330$ & $12.3 \mathrm{ab}$ & 11.8 abcd & $14.7 \mathrm{bc}$ & 2.3 bcde & 3.0 defg & $1.6 \mathrm{abc}$ & $6.0 \mathrm{a}$ & $0.7 \mathrm{a}$ & $0.5 \mathrm{a}$ & $1.3 \mathrm{ab}$ \\
\hline $01 / 1610$ & $12.9 \mathrm{ab}$ & $12.1 \mathrm{abcd}$ & $18.2 \mathrm{abc}$ & 2.2 bcde & 3.8 abcde & $1.4 \mathrm{abc}$ & $7.1 \mathrm{a}$ & $0.5 \mathrm{a}$ & $0.3 \mathrm{a}$ & $1.5 \mathrm{ab}$ \\
\hline $01 / 1331$ & $10.4 \mathrm{ab}$ & $12.1 \mathrm{abcd}$ & $19.3 \mathrm{abc}$ & $2.8 \mathrm{ab}$ & $3.1 \mathrm{cdefg}$ & $1.1 \mathrm{abc}$ & $7.6 \mathrm{a}$ & $0.7 \mathrm{a}$ & $0.4 \mathrm{a}$ & $1.2 \mathrm{ab}$ \\
\hline $95 / 0379$ & $11.2 \mathrm{ab}$ & $12.5 \mathrm{abcd}$ & $9.2 \mathrm{c}$ & $2.1 \mathrm{bcde}$ & $3.3 \mathrm{cdefg}$ & $1.3 \mathrm{abc}$ & $7.2 \mathrm{a}$ & $0.5 \mathrm{a}$ & $0.5 \mathrm{a}$ & $1.2 \mathrm{ab}$ \\
\hline $01 / 1371$ & $9.4 \mathrm{ab}$ & $10.3 \mathrm{~cd}$ & $14.7 \mathrm{bc}$ & $2.1 \mathrm{bcde}$ & 3.5 bcdef & $1.5 \mathrm{abc}$ & $6.1 \mathrm{a}$ & $0.5 \mathrm{a}$ & $0.4 \mathrm{a}$ & $1.5 \mathrm{ab}$ \\
\hline $01 / 1277$ & $10.2 \mathrm{ab}$ & $11.3 \mathrm{abcd}$ & $16.0 \mathrm{bc}$ & $2.3 \mathrm{bcde}$ & $2.6 \mathrm{efg}$ & $1.4 \mathrm{abc}$ & $6.8 \mathrm{a}$ & $0.7 \mathrm{a}$ & $0.3 \mathrm{a}$ & $1.2 \mathrm{ab}$ \\
\hline $01 / 1412$ & $11.5 \mathrm{ab}$ & $11.4 \mathrm{abcd}$ & $14.7 \mathrm{bc}$ & $2.7 \mathrm{abcd}$ & $2.6 \mathrm{efg}$ & $0.9 c$ & $5.1 \mathrm{a}$ & $0.6 \mathrm{a}$ & $0.3 \mathrm{a}$ & $1.4 \mathrm{ab}$ \\
\hline $01 / 1649$ & $11.2 \mathrm{ab}$ & $9.8 \mathrm{~cd}$ & $14.7 \mathrm{bc}$ & $2.1 \mathrm{bcde}$ & 3.0 cdefg & $1.3 \mathrm{abc}$ & $5.6 \mathrm{a}$ & $0.4 \mathrm{a}$ & $0.3 \mathrm{a}$ & $1.3 \mathrm{ab}$ \\
\hline $01 / 1380$ & $8.4 \mathrm{ab}$ & $10.5 \mathrm{~cd}$ & $10.3 \mathrm{c}$ & 2.2 bcde & $3.5 \mathrm{bcdef}$ & $1.2 \mathrm{abc}$ & $5.0 \mathrm{a}$ & $0.4 \mathrm{a}$ & $0.3 \mathrm{a}$ & $1.3 \mathrm{ab}$ \\
\hline $01 / 1235$ & $12.1 \mathrm{ab}$ & $8.9 \mathrm{~cd}$ & $11.4 \mathrm{c}$ & 2.0 bcde & $2.0 \mathrm{~g}$ & $1.0 \mathrm{bc}$ & $5.2 \mathrm{a}$ & $0.7 \mathrm{a}$ & $0.5 \mathrm{a}$ & $1.1 \mathrm{ab}$ \\
\hline $98 / 2132$ & $8.0 \mathrm{ab}$ & 10.9 bcd & $15.1 \mathrm{bc}$ & 2.1 bcde & 3.3 cdefg & $1.4 \mathrm{abc}$ & $4.2 \mathrm{a}$ & $0.4 \mathrm{a}$ & $0.3 \mathrm{a}$ & $1.1 \mathrm{ab}$ \\
\hline $01 / 1206$ & $8.4 \mathrm{ab}$ & $12.5 \mathrm{abcd}$ & $16.1 \mathrm{bc}$ & 1.8 bcde & $2.3 \mathrm{fg}$ & $1.0 \mathrm{bc}$ & $5.6 \mathrm{a}$ & $0.6 \mathrm{a}$ & $0.3 \mathrm{a}$ & $1.2 \mathrm{ab}$ \\
\hline $01 / 1404$ & $8.0 \mathrm{ab}$ & $10.9 \mathrm{bcd}$ & $15.6 \mathrm{bc}$ & 2.1 bcde & 2.9 defg & $1.1 \mathrm{abc}$ & $4.7 \mathrm{a}$ & $0.4 \mathrm{a}$ & $0.3 \mathrm{a}$ & $1.6 \mathrm{ab}$ \\
\hline $01 / 1368$ & $10.1 \mathrm{ab}$ & $9.9 \mathrm{~cd}$ & $11.2 \mathrm{c}$ & 1.8 bcde & 3.1 cdefg & $1.2 \mathrm{abc}$ & $4.3 \mathrm{a}$ & $0.3 \mathrm{a}$ & $0.3 \mathrm{a}$ & $1.7 \mathrm{ab}$ \\
\hline $01 / 1224$ & $9.7 \mathrm{ab}$ & $9.7 \mathrm{~cd}$ & $14.3 \mathrm{bc}$ & 1.8 bcde & 3.1 cdefg & $1.1 \mathrm{abc}$ & $5.1 \mathrm{a}$ & $0.4 \mathrm{a}$ & $0.3 \mathrm{a}$ & $1.1 \mathrm{ab}$ \\
\hline $01 / 1646$ & $7.9 \mathrm{ab}$ & $10.0 \mathrm{~cd}$ & $15.4 \mathrm{bc}$ & $1.9 \mathrm{bcde}$ & $2.5 \mathrm{efg}$ & $1.6 \mathrm{abc}$ & $4.5 \mathrm{a}$ & $0.4 \mathrm{a}$ & $0.3 \mathrm{a}$ & $1.2 \mathrm{ab}$ \\
\hline $94 / 0006$ & $8.1 \mathrm{ab}$ & $9.6 \mathrm{~cd}$ & $8.9 c$ & $1.3 \mathrm{e}$ & 3.2 cdefg & $1.6 \mathrm{abc}$ & $5.0 \mathrm{a}$ & $0.5 \mathrm{a}$ & $0.3 \mathrm{a}$ & $1.0 \mathrm{~b}$ \\
\hline $90 / 01554$ & $7.6 \mathrm{~b}$ & $8.4 \mathrm{~d}$ & $13.4 \mathrm{bc}$ & 2.0 bcde & $2.4 \mathrm{fg}$ & $1.1 \mathrm{abc}$ & $4.2 \mathrm{a}$ & $0.4 \mathrm{a}$ & $0.3 \mathrm{a}$ & $1.1 \mathrm{ab}$ \\
\hline $96 / 1089 \mathrm{~A}$ & $7.6 \mathrm{~b}$ & $10.5 \mathrm{~cd}$ & $11.3 \mathrm{c}$ & $1.5 \mathrm{de}$ & $2.3 \mathrm{fg}$ & $1.1 \mathrm{abc}$ & $3.5 \mathrm{a}$ & $0.3 \mathrm{a}$ & $0.3 \mathrm{a}$ & $1.2 \mathrm{ab}$ \\
\hline 30,572(chk) & $9.4 \mathrm{ab}$ & $9.1 \mathrm{~cd}$ & $9.5 \mathrm{c}$ & $1.6 \mathrm{de}$ & 2.7 defg & $1.0 \mathrm{bc}$ & $4.2 \mathrm{a}$ & $0.4 \mathrm{a}$ & $0.4 \mathrm{a}$ & $1.2 \mathrm{ab}$ \\
\hline TME 1(chk) & $7.7 \mathrm{ab}$ & $7.6 \mathrm{~d}$ & 13.2 bc & 1.6 cde & 2.7 defg & $1.1 \mathrm{bc}$ & $3.4 \mathrm{a}$ & $0.2 \mathrm{a}$ & $0.3 \mathrm{a}$ & $1.6 \mathrm{ab}$ \\
\hline 91/02324(chk) & $8.2 \mathrm{ab}$ & $9.0 \mathrm{~cd}$ & $10.3 \mathrm{c}$ & 1.8 bcde & $4.9 a^{\circ}$ & $1.6 \mathrm{abc}$ & $3.7 \mathrm{a}$ & $0.5 \mathrm{a}$ & $0.4 \mathrm{a}$ & $1.1 \mathrm{ab}$ \\
\hline Minimum & 2.6 & 3.4 & 1.6 & 0.6 & 0.9 & 0.5 & 0.2 & 0.1 & 0.3 & 1 \\
\hline Maximum & 48.9 & 30.9 & 57.8 & 7.1 & 8 & 5.3 & 25 & 5.5 & 4.4 & 9.2 \\
\hline Mean & 10.4 & 11.1 & 14.7 & 2.2 & 3.2 & 1.3 & 5.8 & 0.5 & 0.4 & 1.3 \\
\hline $\begin{array}{l}\text { Standard } \\
\text { deviation }\end{array}$ & 5.5 & 4.4 & 8.3 & 0.8 & 1.4 & 0.6 & 4.1 & 0.5 & 0.3 & 0.9 \\
\hline $\begin{array}{c}\operatorname{Pr}> \\
\text { F(Genotype })\end{array}$ & $* * *$ & $* * *$ & $* * *$ & $* * *$ & $* * *$ & $* * *$ & $* *$ & ns & ns & ns \\
\hline
\end{tabular}

$* * *, * *$, Significant at $p \leq 0.001$ and $p \leq 0.01$ respectively; ns -not significant $p>0.05$. Means with different same letters within the same column are significantly different.

The Fe and $\mathrm{Zn}$ content of the yellow-fleshed cassava is comparable with some raw vegetables and even higher for some genotypes [2]. Interestingly, macro element concentrations for $\mathrm{K}, \mathrm{Ca}$, and $\mathrm{Na}$ in some yellow-fleshed cassava genotypes surpass values reported for popular cereals and soybean, a tested and trusted legume [2]. Fe and $\mathrm{Zn}$ are essential micronutrients for children and adults. Iron helps the human body in the creation of new red blood cells, and together with $\mathrm{Cu}$, boosts red blood cells, thereby preventing anaemia [3]. Zn, on the other hand, strengthens the immune system, assists in protein synthesis, boosts gene expression, aids wound healing, among many more benefits. In Nigeria, about 75 percent of the preschool children and 67 percent of pregnant women are reported to be anemic, and 20 percent of children below five years suffer from zinc deficiency [28]. K plays an important role in the tuberization of cassava because it uses it as a primary osmolyte to osmotically adjust and 
positively respond to water stress [29]. Therefore, this accounts for its abundance in cassava roots. K is a vasodilator due to its ability to reduce strain on the cardiovascular system. Also, it maintains fluid balance in the body for efficient metabolic activities. From the results, all the yellow-fleshed cassava genotypes will convincingly combat diseases brought about by deficiencies of these essential minerals, which are very low in the white varieties commonly consumed.

Table 4. Mean value of macro elements of yellow-fleshed cassava roots by genotypes and across five growing locations

\begin{tabular}{|c|c|c|c|c|c|c|}
\hline Genotype & $\mathrm{Ca}(\mathrm{mg} / \mathrm{kg})$ & $\mathrm{Mg}(\mathrm{mg} / \mathrm{kg})$ & $\mathrm{Na}(\mathrm{mg} / \mathrm{kg})$ & K (mg/kg) & P (mg/kg) & $\mathrm{S}(\mathrm{mg} / \mathrm{kg})$ \\
\hline $01 / 1442$ & 2060.0 abcde & $2517.0 \mathrm{abc}$ & $410.4 \mathrm{ab}$ & $12,690.0$ abcde & $1121.0 \mathrm{ab}$ & $384.0 \mathrm{ab}$ \\
\hline $01 / 1115$ & 1665.0 abcdef & $2236.0 \mathrm{abcd}$ & $285.0 \mathrm{ab}$ & $14,850.0 \mathrm{abc}$ & $959.0 \mathrm{ab}$ & $412.0 \mathrm{a}$ \\
\hline $01 / 1610$ & 1328.0 abcdef & $2612.0 \mathrm{ab}$ & $138.4 \mathrm{ab}$ & 10,240.0 abcde & $1097.0 \mathrm{ab}$ & $384.0 \mathrm{ab}$ \\
\hline $01 / 1273$ & $2388.0 \mathrm{ab}$ & $2972.0 \mathrm{a}$ & $567.7 \mathrm{a}$ & $15,780.0 \mathrm{a}$ & $873.0 \mathrm{~b}$ & $236.0 \mathrm{de}$ \\
\hline 01/1331 & 1795.9 abcdef & 1798.6 abcde & $100.3 \mathrm{~b}$ & $15,006.9 \mathrm{abc}$ & $1407.4 \mathrm{a}$ & 297.9 abcde \\
\hline 01/1371 & $2591.0 \mathrm{a}$ & 1921.0 abcde & $356.0 \mathrm{ab}$ & $11,720.0$ abcde & $849.0 \mathrm{~b}$ & 289.2 bcde \\
\hline $01 / 1663$ & 2176.0 abcde & $2254.0 \mathrm{abcd}$ & $112.4 \mathrm{ab}$ & $15,610.0 \mathrm{ab}$ & $903.0 \mathrm{~b}$ & $319.0 \mathrm{abcd}$ \\
\hline $01 / 1335$ & $2246.0 \mathrm{abcd}$ & 1230.0 cde & $102.9 \mathrm{~b}$ & $14,230.0 \mathrm{abcd}$ & $1109.0 \mathrm{ab}$ & $331.0 \mathrm{abcd}$ \\
\hline $01 / 1404$ & 1538.0 abcdef & 1749.0 abcde & $221.5 \mathrm{ab}$ & $11,390.0$ abcde & $991.0 \mathrm{ab}$ & 285.2 bcde \\
\hline $01 / 1413$ & 1519.0 abcdef & 1870.0 abcde & $213.4 \mathrm{ab}$ & 9910.0 abcde & $990.0 \mathrm{ab}$ & $318.0 \mathrm{abcd}$ \\
\hline $01 / 1649$ & 1096.0 bcdef & 1473.0 bcde & $90.3 \mathrm{~b}$ & $11,510.0$ abcde & $964.0 \mathrm{ab}$ & $331.0 \mathrm{abcd}$ \\
\hline $01 / 1224$ & $2379.0 \mathrm{abc}$ & 1185.0 de & $125.2 \mathrm{ab}$ & 8850.0 cde & $790.0 \mathrm{~b}$ & $332.0 \mathrm{abcd}$ \\
\hline $01 / 1380$ & 1019.0 def & 1686.0 abcde & $134.8 \mathrm{ab}$ & 9390.0 abcde & $945.0 \mathrm{~b}$ & $320.0 \mathrm{abcd}$ \\
\hline $90 / 01554$ & 1909.0 abcdef & 1498.0 bcde & $164.5 \mathrm{ab}$ & 8320.0 de & $920.0 \mathrm{~b}$ & 271.6 cde \\
\hline $01 / 1277$ & 1684.0 abcdef & 1433.0 bcde & $203.7 \mathrm{ab}$ & $11,530.0$ abcde & $862.0 \mathrm{~b}$ & 231.2 de \\
\hline $95 / 0379$ & 1704.0 abcdef & 1425.0 bcde & $119.2 \mathrm{ab}$ & $10,100.0$ abcde & $948.0 \mathrm{ab}$ & 253.7 cde \\
\hline $94 / 0330$ & 1550.0 abcdef & 1521.0 bcde & $87.3 \mathrm{~b}$ & $7660.0 \mathrm{e}$ & $1106.0 \mathrm{ab}$ & 281.0 bcde \\
\hline $01 / 1412$ & 1936.0 abcdef & 1498.0 bcde & $100.1 \mathrm{~b}$ & $12,580.0$ abcde & $835.0 \mathrm{~b}$ & 253.5 cde \\
\hline $01 / 1368$ & 1175.0 bcdef & 1500.0 bcde & $187.9 \mathrm{ab}$ & 8660.0 cde & $883.0 \mathrm{~b}$ & 280.4 bcde \\
\hline $98 / 2132$ & 1257.0 bcdef & 1182.0 de & $174.8 \mathrm{ab}$ & 9200.0 bcde & $962.0 \mathrm{ab}$ & 275.1 cde \\
\hline 96/1089A & 1642.0 abcdef & $1028.0 \mathrm{de}$ & $119.7 \mathrm{ab}$ & 8670.0 cde & $911.0 \mathrm{~b}$ & 280.2 bcde \\
\hline $01 / 1646$ & 1743.0 abcdef & 1355.0 bcde & $57.4 \mathrm{~b}$ & $10,060.0$ abcde & $881.0 \mathrm{~b}$ & 249.8 cde \\
\hline $01 / 1235$ & 1076.0 cdef & 1571.0 bcde & $81.1 \mathrm{~b}$ & $10,040.0$ abcde & $783.0 \mathrm{~b}$ & 288.8 bcde \\
\hline $01 / 1206$ & 1726.0 abcdef & 1065.0 de & $102.8 \mathrm{~b}$ & 9980.0 abcde & $881.0 \mathrm{~b}$ & $208.5 \mathrm{e}$ \\
\hline $94 / 0006$ & 938.0 ef & $1045.0 \mathrm{de}$ & $59.8 \mathrm{~b}$ & 8220.0 de & $1018.0 \mathrm{ab}$ & 304.4 abcde \\
\hline 91/02324(chk) & 1111.0 bcdef & $763.0 \mathrm{e}$ & $106.6 \mathrm{ab}$ & 9600.0 abcde & $1094.0 \mathrm{ab}$ & $350.0 \mathrm{abc}$ \\
\hline 30572(chk) & 1094.0 bcdef & $874.0 \mathrm{e}$ & $131.6 \mathrm{ab}$ & $6670.0 \mathrm{e}$ & $952.0 \mathrm{ab}$ & 287.6 bcde \\
\hline TME 1(chk) & $723.0 \mathrm{f}$ & $796.0 \mathrm{e}$ & $69.0 \mathrm{~b}$ & 7750.0 de & $1072.0 \mathrm{ab}$ & 254.9 cde \\
\hline Minimum & 410 & 460 & 4 & 2100 & 450 & 138 \\
\hline Maximum & 7600 & 8100 & 4100 & 43,000 & 3100 & 700 \\
\hline Mean & 1604 & 1576 & 167 & 10,637 & 967 & 297 \\
\hline Std. deviation & 1057 & 1077 & 297 & 7172 & 458 & 94 \\
\hline $\begin{array}{c}\operatorname{Pr}> \\
\mathrm{F}(\text { Genotype })\end{array}$ & $* * *$ & $* * *$ & * & $* * *$ & $* *$ & $* * *$ \\
\hline
\end{tabular}

Most of the yellow-fleshed genotypes had higher values of the trace elements than the three white-fleshed genotypes used as check samples (Table 4). This observation shows that the yellow-fleshed cassava roots compared favorably with the white-fleshed cassava roots in terms of microelement content, and it confers an additional nutritional benefit to the yellow-fleshed roots. There were no significant $(p>0.05)$ differences among the genotypes for $\mathrm{Al}, \mathrm{Ti}, \mathrm{Cr}$, and $\mathrm{Pb}$. It bolsters the earlier observation that genotype did not play a significant influence on these trace elements. However, they are all $<10.0 \mathrm{mg} / 100 \mathrm{~g}$, unlike the $\mathrm{Fe}, \mathrm{Zn}$, and $\mathrm{Mn}$ that had higher values. The respective genotypes had the highest Fe (01/1442), Zn (01/1442), Mn (01/1442), B (01/1442), Cu (01/1442), Ni (01/1115), Al (01/1442), $\mathrm{Ti}$ (01/1442), $\mathrm{Cr}(01 / 1413)$, and $\mathrm{Pb}$ (01/1163) content, while the respective genotypes with the lowest values were: Fe (90/01554, 96/1089A), Zn (90/01554), Mn (94/006), B (94/006), Cu (01/1235), Ni (01/1412), $\mathrm{Al}$ (96/1089A), $\mathrm{Ti}(01 / 1368)$, and $\mathrm{Pb}$ (94/006). Genotype 01/1442 was outstanding because it showed 
the highest values for almost all the minerals studied. However, genotypes 90/01554 and 90/1089A were the poor ones because they had the lowest amounts of Fe and $\mathrm{Zn}$, the essential micronutrient in terms of their nutritional importance. Genotype 01/1442 is a potential pipeline genotype that could be advanced for release as a high micronutrient cassava variety. It will help to address the micronutrient deficiencies in the cassava growing and consuming communities of Nigeria.

The macro element content of yellow-fleshed cassava roots by genotype shows a general increase in values as compared to white-fleshed cassava roots. There were no significant differences among the genotypes for all the elements at a $5 \%$ significance level. However, these genotypes have the highest $\mathrm{Ca}(01 / 1371), \mathrm{Mg}$ (01/1273), $\mathrm{Na}(01 / 1273), \mathrm{K}$ (01/1273), P (01/1331), S (01/1115), while the respective genotypes exhibit the lowest $\mathrm{Ca}(94 / 0006), \mathrm{Mg}(96 / 1089 \mathrm{~A}), \mathrm{Na}(01 / 1646), \mathrm{K}(94 / 0330)$, $\mathrm{P}(01 / 1235)$, and S (01/1206). Genotype 01/1273 showed the highest values for half of the elements under observation, while no genotype can be qualified as inferior because none has low value across all elements. Ref. [30] reported there was no difference in concentrations of Fe and $\mathrm{Zn}$ in 10 cassava genotypes planted in two growing locations, namely, Nampula and Luipo. However, differences were significant at another growing location known as Umbeluzi with values for Fe $(p<0.001)$ and $\mathrm{Zn}(p=0.0162)$ ranging from $8-24 \mathrm{mg} / \mathrm{kg}$ and from $8-19 \mathrm{mg} / \mathrm{kg}$, respectively. These reported values are comparable with results in this research, although high values such as the ones reported were not recorded. This follows the same trend as observed that the growing locations have a highly significant effect $(p<0.05)$ in the trace element concentration of yellow-fleshed cassava (Table 5). Akin-Idowu et al. [31] reported a similar trend where eight yam genotypes were planted in two growing locations (Ibadan and Onne).

Table 5. Mean value of trace and macro elements of yellow-fleshed cassava roots by growing locations

\begin{tabular}{|c|c|c|c|c|c|c|c|c|c|c|}
\hline \multirow{2}{*}{$\begin{array}{l}\text { Growing } \\
\text { Locations }\end{array}$} & \multicolumn{10}{|c|}{ Parameters (mg/kg) } \\
\hline & $\mathrm{Fe}$ & $\mathrm{Zn}$ & Mn & B & $\mathrm{Cu}$ & $\mathbf{N i}$ & Al & Ti & $\mathrm{Cr}$ & $\mathrm{Pb}$ \\
\hline Mokwa & $12.8 \mathrm{a}$ & $11.3 \mathrm{~b}$ & $22.1 \mathrm{a}$ & $2.3 \mathrm{a}$ & $2.1 \mathrm{~d}$ & $1.6 \mathrm{a}$ & $6.4 \mathrm{a}$ & $0.6 \mathrm{a}$ & $0.4 \mathrm{a}$ & $1.5 \mathrm{~b}$ \\
\hline Zaria & $10.7 \mathrm{ab}$ & $15.6 \mathrm{a}$ & $19.2 \mathrm{a}$ & $2.3 \mathrm{ab}$ & $2.7 \mathrm{c}$ & $1.6 \mathrm{a}$ & $6.2 \mathrm{a}$ & $0.4 \mathrm{a}$ & $0.3 \mathrm{a}$ & $2.1 \mathrm{a}$ \\
\hline Ibadan & $12.5 \mathrm{a}$ & $10.6 \mathrm{~b}$ & $11.0 \mathrm{bc}$ & $2.2 \mathrm{ab}$ & $3.7 \mathrm{~b}$ & $1.5 \mathrm{a}$ & $7.2 \mathrm{a}$ & $0.5 \mathrm{a}$ & $0.4 \mathrm{a}$ & $1.0 \mathrm{c}$ \\
\hline Ubiaja & $8.7 \mathrm{bc}$ & $6.8 \mathrm{c}$ & $12.5 \mathrm{~b}$ & $2.1 \mathrm{ab}$ & $2.8 \mathrm{c}$ & $1.0 \mathrm{~b}$ & $5.4 \mathrm{ab}$ & $0.5 \mathrm{a}$ & $0.4 \mathrm{a}$ & $1.1 \mathrm{c}$ \\
\hline Onne & $7.6 \mathrm{c}$ & $11.2 \mathrm{~b}$ & $8.9 c$ & $1.9 \mathrm{~b}$ & $4.6 \mathrm{a}$ & $0.8 \mathrm{~b}$ & $3.9 \mathrm{~b}$ & $0.5 \mathrm{a}$ & $0.3 \mathrm{a}$ & $1.0 \mathrm{C}$ \\
\hline $\begin{array}{c}\operatorname{Pr}>\mathrm{F} \\
\text { (Locations) }\end{array}$ & $* * *$ & $* * *$ & $* * *$ & $* *$ & $* * *$ & $* * *$ & $* *$ & ns & ns & $* * *$ \\
\hline \multirow{2}{*}{$\begin{array}{l}\text { Growing } \\
\text { Locations }\end{array}$} & \multicolumn{10}{|c|}{ Parameters (mg/kg) } \\
\hline & $\mathrm{Ca}$ & \multicolumn{2}{|c|}{$\mathrm{Mg}$} & $\mathrm{Na}$ & \multicolumn{2}{|c|}{$\mathbf{K}$} & \multicolumn{2}{|c|}{$\mathbf{P}$} & \multicolumn{2}{|c|}{$S$} \\
\hline Onne & $1220.9 \mathrm{~b}$ & \multicolumn{2}{|c|}{$1816.1 \mathrm{~b}$} & $247.7 \mathrm{a}$ & \multicolumn{2}{|c|}{$4323.2 \mathrm{c}$} & \multicolumn{2}{|c|}{$1668.2 \mathrm{a}$} & \multicolumn{2}{|c|}{$388.7 \mathrm{a}$} \\
\hline Mokwa & $2345.4 \mathrm{a}$ & \multicolumn{2}{|c|}{$1945.7 \mathrm{ab}$} & $182.3 \mathrm{ab}$ & \multicolumn{2}{|c|}{$14,408.9 \mathrm{~b}$} & \multicolumn{2}{|c|}{$840.2 \mathrm{~b}$} & \multicolumn{2}{|c|}{$221.0 \mathrm{c}$} \\
\hline Ubiaja & $1059.1 \mathrm{~b}$ & \multicolumn{2}{|c|}{$2257.5 \mathrm{a}$} & $276.0 \mathrm{a}$ & \multicolumn{2}{|c|}{$3769.6 \mathrm{c}$} & \multicolumn{2}{|c|}{$924.5 \mathrm{~b}$} & \multicolumn{2}{|c|}{$292.3 \mathrm{~b}$} \\
\hline Zaria & $2258.0 \mathrm{a}$ & \multicolumn{2}{|c|}{$967.8 \mathrm{c}$} & $34.5 \mathrm{c}$ & \multicolumn{2}{|c|}{$18,199.4 \mathrm{a}$} & \multicolumn{2}{|c|}{$612.6 \mathrm{c}$} & \multicolumn{2}{|c|}{$288.5 \mathrm{~b}$} \\
\hline Ibadan & $1164.6 \mathrm{~b}$ & \multicolumn{2}{|c|}{$880.4 \mathrm{c}$} & 85.1 bc & \multicolumn{2}{|c|}{$12,908.9 \mathrm{~b}$} & \multicolumn{2}{|c|}{$795.0 \mathrm{~b}$} & \multicolumn{2}{|c|}{$293.4 \mathrm{~b}$} \\
\hline $\begin{array}{c}\operatorname{Pr}>\mathrm{F} \text { (Growing } \\
\text { locations) }\end{array}$ & $* * *$ & \multicolumn{2}{|c|}{$* * *$} & $* * *$ & \multicolumn{2}{|c|}{$* * *$} & \multicolumn{2}{|c|}{$* * *$} & \multicolumn{2}{|c|}{$* * *$} \\
\hline
\end{tabular}

$* * *, * *$ Significant at $p \leq 0.001$ and $p \leq 0.01$ respectively; ns—not significant $p>0.05$. Means with different same letters within the same column are significantly different.

There was a significant $(p<0.05)$ difference between the mineral content of the samples between the growing locations. It can be observed that Mokwa, Zaria, and Ibadan growing locations have higher concentrations for all trace elements except for Onne that records the highest concentration of $\mathrm{Cu}$ (Table 5). In the case of the macro elements, Ubiaja and Onne locations had higher values for all the elements except $\mathrm{K}$. The variations of the mineral content further established that mineral content could fluctuate greatly depending on genetic and climatic factors, agricultural procedures, the composition of the soil, and ripeness of the harvested crops, among other factors. This applies to both macro and trace elements. However, changes in the mineral content usually occur also in the processing of raw 
materials, e.g., in thermal processes and material separation, which determine the available mineral content in the cassava products.

3.3. Principal Component (PC) and Cluster Analysis of the Trace and Macroelements of Yellow-Fleshed Cassava Roots Across Five Growing Locations

Table 6 showed the eigenvector of the first three axes of the Principal components (PCs) of the trace elements of yellow-fleshed cassava roots across the genotypes and growing locations. Four PCs (PC1 to 4) explained $75.2 \%$ of the data, but PC1 and PC2 accounted for above $50 \%$, and PC1 had the highest eigenvalue of 3.5, while PC2 and PC3 had only 1.5 and 1.4, respectively. The minerals with common characteristics were loaded on each of the PCs. Thus, Fe, Mn, B, and Al were on PC1, Ti, $\mathrm{Pb}$, and $\mathrm{Mn}$ on $\mathrm{PC} 2$, while $\mathrm{Zn}, \mathrm{Cu}$, and $\mathrm{Cr}$ were loaded on $\mathrm{PC} 3$. There was a positive and significant $(p<0.05)$ correlation among all the minerals on PC1, a positive and significant $(p<0.05)$ correlation between $\mathrm{Mn}$ and $\mathrm{Pb}$. At the same time, both have a significant $(p<0.05)$ negative correlation with Ti on PC2. On PC3, Zn and Cu correlate negatively with $\mathrm{Cr}$. In a recent study, $\mathrm{Mn}$ and $\mathrm{B}$; and $\mathrm{K}$ and $\mathrm{P}$ were reported to be positively correlated [15]. There is a similar observation for Mn and B, but a negative correlation was observed for $\mathrm{K}$ and $\mathrm{P}$ in this study.

Table 6. Eigenvectors of the first three axes of the principal components (PC) analysis of the trace and macro elements of yellow-fleshed cassava roots

\begin{tabular}{|c|c|c|c|}
\hline Parameters & PC1 & PC2 & PC3 \\
\hline $\mathrm{Fe}$ & $0.4^{* * *}$ & - & \\
\hline $\mathrm{Zn}$ & - & - & $-0.4^{* * *}$ \\
\hline $\mathrm{Mn}$ & $0.4^{* * *}$ & $0.4^{* * *}$ & - \\
\hline B & $0.4^{* * *}$ & - & - \\
\hline $\mathrm{Cu}$ & - & - & $-0.5^{* * *}$ \\
\hline $\mathrm{Ni}$ & - & - & - \\
\hline $\mathrm{Al}$ & $0.4^{* * *}$ & - & - \\
\hline $\mathrm{Ti}$ & - & $-0.4^{* * *}$ & - \\
\hline $\mathrm{Cr}$ & - & - & $0.5^{* * *}$ \\
\hline $\mathrm{Pb}$ & - & $0.6^{* * *}$ & - \\
\hline Eigenvalue & 3.5 & 1.5 & 1.4 \\
\hline Cumulative \% & 35.2 & 50.5 & 64.1 \\
\hline Parameters & PC1 & PC2 & PC3 \\
\hline $\mathrm{Ca}$ & - & $0.6^{* * *}$ & 0.4 \\
\hline $\mathrm{Mg}$ & $0.4^{* * *}$ & $0.5^{* * *}$ & - \\
\hline $\mathrm{Na}$ & $0.4^{* * *}$ & $0.5^{* *}$ & - \\
\hline $\mathrm{K}$ & $-0.5^{* * *}$ & - & $0.5^{* *}$ \\
\hline $\mathrm{P}$ & $0.5^{* * *}$ & - & $0.4^{* *}$ \\
\hline$S$ & 0.4 & - & 0.6 \\
\hline Eigenvalue & 2 & 1.7 & 1.1 \\
\hline Cumulative \% & 34.1 & 61.9 & 80.3 \\
\hline
\end{tabular}

Furthermore, the principal component analysis for macroelements (PC1 to 3) explained the $80.3 \%$ of the data with PC1, having the highest eigenvalue of 2.0, PC2 1.7, and PC3 1.1. The macro elements contributing to $\mathrm{PC} 1$ are $\mathrm{Mg}, \mathrm{Na}, \mathrm{K}, \mathrm{P}$, and $\mathrm{S}$, with $\mathrm{K}$ having a significant negative correlation with the others. Macro elements contributing to $\mathrm{PC} 2$ are $\mathrm{Ca}, \mathrm{Mg}$, and $\mathrm{Na}$, all having significant positive correlations with one another. $\mathrm{Ca}, \mathrm{K}, \mathrm{P}$, and $\mathrm{S}$ are loadings on $\mathrm{PC} 3$, having only $\mathrm{K}$ and $\mathrm{P}$ correlating significantly, while $\mathrm{Ca}$ and $\mathrm{S}$ have no significance. A similar result of negative correlation between $\mathrm{Na}$ and $\mathrm{K}$ in yellow-fleshed cassava root was reported by [15]. Thus, the macro elements in their respective loadings are related together, which will help in breeding for suitable agronomic traits with the desired macro element(s) in the yellow-fleshed cassava genotypes under consideration. It has been established 
that macro elements have been absorbed more than trace elements by the stem and root crops [19,32], which is evident from their principal components. However, across the five growing locations, a highly significant difference is still observed for both trace and macro elements, which proves beyond a reasonable doubt that a breeder will have clarity in making decisions involving trace and macro elements as regards growing locations.

\subsection{Multivariate Cluster Analysis}

Table 7 and Figure 1 show a cluster of the yellow-fleshed cassava roots genotypes based on their dissimilarity for the 16 quantitative mineral traits investigated. This produced three groups of genotypes across all growing locations. Using the Euclidean distance for dissimilarity and Ward's method as the agglomeration method, show specific portions of variance explained by each of the trait (Figure 1). Cluster 1 had 12 yellow-fleshed genotypes with two white-fleshed varieties (TME1 and 91/02324) used as checks. It implies that these genotypes compared well with the two check varieties. Cluster 2 had eight yellow-fleshed genotypes, and Cluster 3 had five yellow-fleshed genotypes and one white-fleshed check variety $(30,572)$. It was observed that the genotypes identified with the highest trace element concentration (01/1442) and macroelement concentration (01/1273) clustered in the same group (2) with genotype $01 / 1273$ on top of the group while genotype $01 / 1442$ is at the bottom. The genotypes identified as the poor ones (90/01554) and (96/1089A) with the lowest Fe and Zn are in groups 3 and 1, respectively. This strongly suggests that cassava genotypes in group 2 contain high concentrations of essential trace and macroelements.

Table 7. Cluster analysis of yellow-fleshed cassava roots across all growing locations using all parameters

\begin{tabular}{cccc}
\hline Cluster & $\mathbf{1}$ & $\mathbf{2}$ & $\mathbf{3}$ \\
\hline & $01 / 1224$ & $01 / 1273$ & $90 / 01554$ \\
$94 / 0006$ & $01 / 1404$ & $01 / 1277$ \\
$94 / 0330$ & $01 / 1412$ & $01 / 1368$ \\
$96 / 1089 \mathrm{~A}$ & $01 / 1335$ & $01 / 1380$ \\
$01 / 1663$ & $01 / 1610$ & $01 / 1235$ \\
$01 / 1115$ & $01 / 1371$ & 30572 \\
$01 / 1413$ & $01 / 1206$ & \\
$98 / 2132$ & $01 / 1442$ & \\
$95 / 0379$ & & \\
$01 / 1646$ & & \\
$91 / 02324$ & & \\
TME 1 & & \\
$01 / 1649$ & & \\
$01 / 1331$ & & \\
\hline
\end{tabular}

Furthermore, none of the check samples, which are white varieties, fell into group 2, two of them were in group 1 and the other one in group 3 . The check samples are typical of the genotypes identified as deficient in minerals compared with pipeline genotypes that are typically referred to as 'improved'. According to the dendrogram, there are several noticeable dissimilarities/differences among genotypes in groups 1, 2, and 3 (Figure 1).

\subsection{Pearson Correlation Coefficients of Trace and Macroelements of Yellow-Fleshed Cassava Roots Genotypes Across Five Growing Locations}

Table 8 shows the Pearson correlation coefficients of trace elements of yellow-fleshed cassava roots genotypes across five growing locations. It can be deduced that $\mathrm{Fe}, \mathrm{Al}, \mathrm{Cr}, \mathrm{Ni}, \mathrm{Mn}, \mathrm{Zn}, \mathrm{B}$, and $\mathrm{Ti}$, have strong significant $(p<0.001)$ correlations across the growing locations while $\mathrm{Zn}, \mathrm{Pb}$. and $\mathrm{Cu}$ have weak $(p<0.05)$ correlation across the growing locations. However, some also exhibit negative correlations, which can be a basis for predicting fertilizer formulation if the need for application arises. The increased concentration of a trace element could lead to the depletion of another; thus, care is needed during a 
breeding program to ensure the purpose of biofortification is not forfeited. In consideration of the macroelements, there are mild to strong correlations across the growing locations, but several negative correlations are observed. S has a significant $(p<0.05)$ negative correlation with $\mathrm{K}$ and $\mathrm{Ca}(\mathrm{r}=-0.154$, $-0.119)$, respectively, but a significant positive $(p<0.001)$ correlation with $p(\mathrm{r}=0.505)$. This correlation shows the possibility of breeding yellow-root cassava varieties with high $\mathrm{K}$ and $p$ mineral content. Also, $\mathrm{K}$ has a significant $(p<0.05$ and $p<0.01)$ negative correlation with $\mathrm{Mg}$ and $\mathrm{Na}(\mathrm{r}=-0.170$ and -0.216 ). However, the negative correlations observed may not be detrimental in real-life situations because of research reports that micronutrients are stable across growing locations. Moreover, it implies that it is feasible to combine the high micronutrient content with high yield in breeding, unlike protein content and yield that are negatively correlated [33].

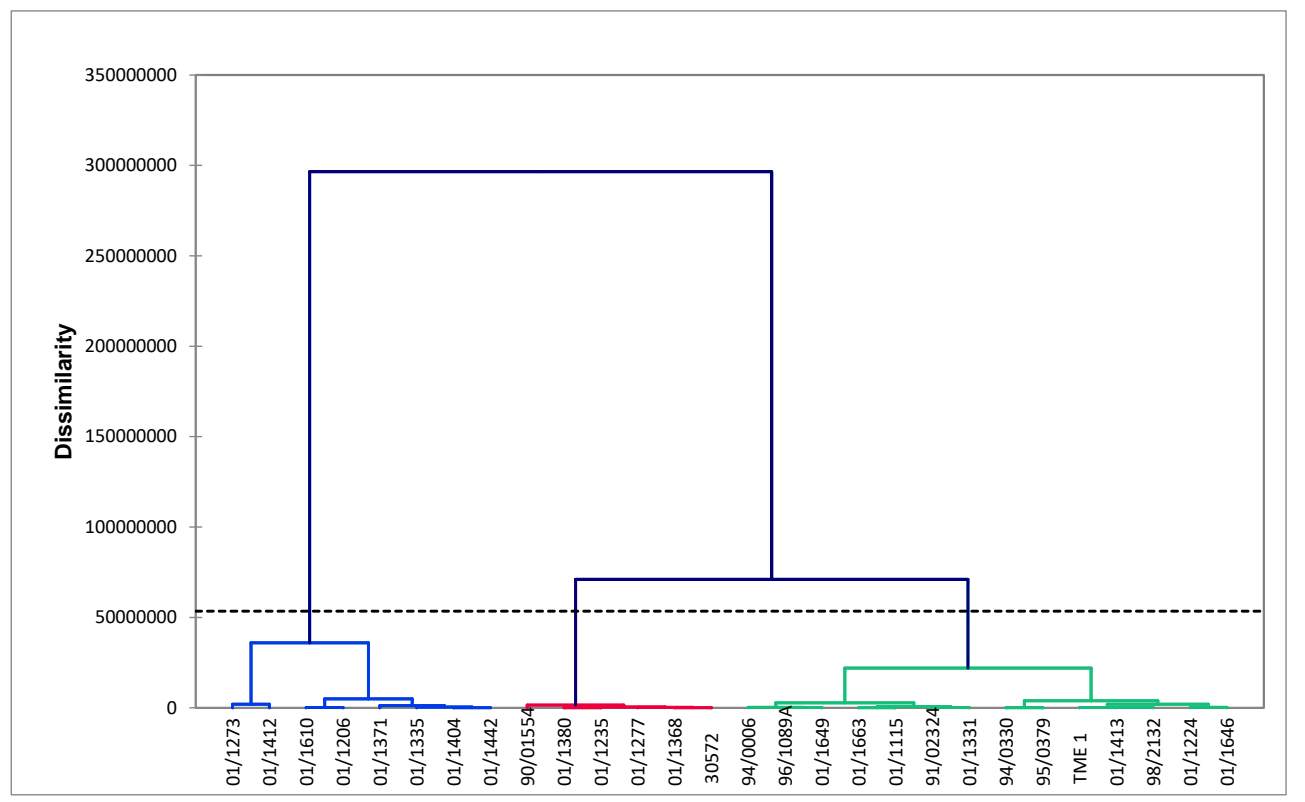

Figure 1. Dendrogram (Ward's minimum variance clusters) showing the relationship among different cassava roots genotypes across growing locations.

Table 8. Pearson correlation coefficients of trace and macro elements of yellow-fleshed cassava roots across genotypes and growing locations

\begin{tabular}{|c|c|c|c|c|c|c|c|c|c|c|}
\hline Parameters & $\mathbf{N i}$ & $\mathrm{Cr}$ & $\mathrm{Fe}$ & Al & $\mathrm{Ti}$ & B & Mn & $\mathrm{Zn}$ & $\mathbf{P b}$ & $\mathrm{Cu}$ \\
\hline $\mathrm{Ni}$ & 1 & & & & & & & & & \\
\hline $\mathrm{Cr}$ & $0.316^{* * * *}$ & 1 & & & & & & & & \\
\hline $\mathrm{Fe}$ & $0.401^{* * *}$ & $0.636^{* * *}$ & 1 & & & & & & & \\
\hline $\mathrm{Al}$ & $0.174^{* *}$ & $0.158^{* *}$ & $0.663 * * *$ & 1 & & & & & & \\
\hline $\mathrm{Ti}$ & 0.09 & $0.165^{* *}$ & $0.527^{* * *}$ & $0.728^{* * *}$ & 1 & & & & & \\
\hline B & 0.054 & $0.186^{* *}$ & $0.426^{* * *}$ & $0.449^{* * *}$ & $0.317^{* * * *}$ & 1 & & & & \\
\hline Mn & $0.350 * * *$ & 0.111 & $0.404^{* * *}$ & $0.394^{* * *}$ & $0.234^{* * *}$ & $0.512 * * *$ & 1 & & & \\
\hline $\mathrm{Zn}$ & $0.287^{* * * *}$ & 0.015 & $0.334^{* * *}$ & $0.310 * * *$ & $0.153 *$ & $0.466^{* * *}$ & $0.503^{* * *}$ & 1 & & \\
\hline $\mathrm{Pb}$ & $0.169 * *$ & -0.016 & $0.123 *$ & 0.116 & 0.001 & 0.048 & $0.426^{* * *}$ & 0.3 & 1 & \\
\hline $\mathrm{Cu}$ & -0.027 & -0.066 & -0.038 & 0.006 & 0.068 & $0.147^{*}$ & $-0.215^{* *}$ & 0.210 ** & $-0.217^{* *}$ & 1 \\
\hline Parameters & $S$ & $\mathrm{P}$ & K & $\mathrm{Ca}$ & $\mathrm{Mg}$ & $\mathrm{Na}$ & & & & \\
\hline$S$ & 1 & & & & & & & & & \\
\hline $\mathrm{P}$ & $0.505^{* * *}$ & 1 & & & & & & & & \\
\hline K & -0.154 * & $\underset{* * *}{-0.327}$ & 1 & & & & & & & \\
\hline $\mathrm{Ca}$ & -0.119 * & $-0.156^{*}$ & $0.531^{* * *}$ & 1 & & & & & & \\
\hline $\mathrm{Mg}$ & 0.086 & $0.263^{* * *}$ & -0.170 * & $0.289 * * *$ & 1 & & & & & \\
\hline $\mathrm{Na}$ & 0.045 & $0.139 *$ & $-0.216^{* *}$ & 0.124 * & $0.597^{* * * *}$ & 1 & & & & \\
\hline
\end{tabular}

$*, * *, * *$ Significant at $p \leq 0.05, p \leq 0.01$ and $p \leq 0.001$ respectively. 


\section{Conclusions}

This study provides information on the concentration of trace and macroelements of yellow-fleshed cassava genotypes based on the interaction between their genetic make-up and the growing locations in which they are planted. It could be concluded that among the explanatory variables, the variable growing locations was the most influential on macro and trace elements. However, genotype had a strong influence on B and Ti. Most of the yellow-fleshed genotypes had higher values of the trace elements than the three white-fleshed genotypes used as check samples. The trace elements that positioned themselves to be the most abundant in yellow-fleshed cassava regardless of the genotype are $\mathrm{Fe}, \mathrm{Mn}$, and $\mathrm{B}$. At the same time, $\mathrm{Mg}, \mathrm{Na}, \mathrm{K}, \mathrm{P}$, and $\mathrm{S}$ showed up as the most abundant for macroelements. The genotypes 01/1273, 01/1404, 01/1412, 01/1335, 01/1610, 01/1371, 01/1206, and 01/1442 were clustered together and identified to be different from the white-fleshed varieties used as checks and were promising pipelines. However, from the group, genotypes 01/1442 and 01/1273 have outstanding trace and macroelement concentrations, respectively. They could be advanced in the breeding cycle as new varieties to combat hidden hunger in Nigeria. However, all genotypes of the yellow-fleshed cassava can be predicted to perform well in any of the growing locations in terms of their trace and macroelement concentrations. The research findings from this study supported the claim that yellow-fleshed cassava roots could be a good source of certain micronutrients and macronutrients capable of combating hidden hunger in most of the cassava growing and consuming areas of the developing countries. However, there is a need for the investigation of the effect of traditional processing and bioavailability of the most important minerals of the identified yellow-fleshed cassava genotypes.

Author Contributions: E.O.A. conceived and drafted the paper; E.O.A. and B.M.-D. were involved with laboratory analysis, data cleaning, analysis, and interpretation; E.O.A., B.M.-D., and A.G.D. contributed to the manuscript by performing a critical interpretation of results and finalized the manuscript. All authors have read and agreed to the published version of the manuscript.

Funding: This research received no external funding

Acknowledgments: The authors acknowledged the supports from the HarvestPlus, CGIAR Research Program on Roots, Tubers and Bananas (RTB), the staff of Food and Nutrition Sciences Laboratory, Cassava Breeding Unit, IITA, Ibadan, Nigeria, and Waite Analytical Services, School of Agriculture and Wine, University of Adelaide, Australia. There is no conflict of interest, and all authors have read and approved the manuscript and aware of its submission.

Conflicts of Interest: All authors declare no conflict of interest.

Data Availability: All data are deposited into CKAN, an IITA data repository, and can be accessed http://data.iita.org/. Also, the data could be made available on request from the corresponding author.

\section{References}

1. Alamu, E.O.; Maziya-Dixon, B.; Dixon, A.G. Evaluation of proximate composition and pasting properties of high-quality cassava flour (HQCF) from cassava genotypes (Manihot esculenta Crantz) of $\beta$-carotene-enriched roots. LWT 2017, 86, 501-506. [CrossRef]

2. Montagnac, J.A.; Davis, C.R.; Tanumihardjo, S.A. Nutritional value of cassava for use as a staple food and recent advances for improvement. Compr. Rev. Food Sci. Food Saf. 2009, 8, 181-194. [CrossRef]

3. Temesgen, Z.T.; Bakalo, B.; Tamirat, T. Medicinal, Nutritional and Anti-Nutritional Properties of Cassava (Manihot esculenta). Acad. J. Nutr. 2019, 8, 34-46. [CrossRef]

4. Chandrasekara, A.; Joseph Kumar, T. Roots, and tuber crops as functional foods: A review on phytochemical constituents and their potential health benefits. Int. J. Food Sci. 2016, 3631647. [CrossRef] [PubMed]

5. Von Grebmer, K.; Saltzman, A.; Birol, E.; Wiesmann, D.; Prasai, N.; Yin, S.; Yohannes, Y.; Menon, P. 2014 Global Hunger Index: The Challenge of Hidden Hunger; International Food Policy Research Institute: Bonn, Germany; Washington, DC, USA; Dublin, Ireland, 2014.

6. Ayetigbo, O.; Latif, S.; Abass, A.; Müller, J. Comparing characteristics of root, flour, and starch of biofortified yellow-flesh and white-flesh cassava variants, and sustainability considerations: A review. Sustainability 2018, 10, 3089. [CrossRef] 
7. Talsma, E.F.; Brouwer, I.D.; Verhoef, H.; Mbera, G.N.K.; Mwangi, A.M.; Demir, A.Y.; Maziya-Dixon, B.; Boy, E.; Zimmermann, M.B.; Melse-Boonstra, A. Biofortified yellow cassava and vitamin A status of Kenyan children: A randomized controlled trial. Am. J. Clin. Nutr. 2016, 103, 258-267. [CrossRef] [PubMed]

8. Belitz, H.D.; Grosch, W.; Schieberle, P. Food Chemistry, 4th ed.; Springer: Berlin/Heidelberg, Germany, 2009; p. 1116. [CrossRef]

9. CÎRŢîNA $\breve{A}$, P.D.; CĂPĂȚîNĂ, C. Considerations on the Role of Mineral Substances in the Organism. Synthesis 2018, 4, 322-326.

10. Gregory, P.J.; Wahbi, A.; Adu-Gyamfi, J.; Heiling, M.; Gruber, R.; Joy, E.J.; Broadley, M.R. Approaches to reduce zinc and iron deficits in food systems. Glob. Food Secur. 2017, 15, 1-10. [CrossRef]

11. Woolfe, J.A.; Woolfe, W.J.; Poats, S.V. The Potato in the Human Diet; Cambridge University Press: Cambridge, UK, 1987; p. 248. ISBN 978-0521112994.

12. Odoom, E. The Potassium Status of Soils and Its Absorption by Cassava in Selected Farms in the Central Region. Ph.D. Thesis, University of Cape Coast, Cape Coast, Ghana, 2017. Available online: http: //hdl.handle.net/123456789/3436 (accessed on 15 March 2020).

13. Mohan, G.; Raju, J.; Shiny, R.; Abhilash, P.V.; Soumya, S. Biochemical, mineral, and proximate composition of Indian cassava varieties. IJCS 2019, 7, 1059-1065.

14. Ghandilyan, A.; Vreugdenhil, D.; Aarts, M.G. Progress in the genetic understanding of plant iron and zinc nutrition. Physiol. Plant. 2006, 126, 407-417. [CrossRef]

15. Alamu, E.O.; Maziya-Dixon, B.; Parkes, E.; Dixon, A.G. Variations of Macro-and Microelements in Yellow-Fleshed Cassava (Manihot esculenta Crantz) Genotypes as a Function of Storage Root Portion, Harvesting Time, and Sampling Method. Appl. Sci. 2020, 10, 5396. [CrossRef]

16. Maziya-Dixon, B.; Alamu, E.O.; Dixon, A.G. Variation in the evaluation of cis-and trans-carotene in yellow-fleshed cassava (Manihot esculenta Cranz) varieties as a function of the storage root portion and sampling method. LWT 2016, 70, 296-301. [CrossRef]

17. Wheal, M.S.; Fowles, T.O.; Palmer, L.T. A cost-effective acid digestion method using closed polypropylene tubes for inductively coupled plasma optical emission spectrometry (ICP-OES) analysis of plant essential elements. Anal. Methods 2011, 3, 2854-2863. [CrossRef]

18. SAS. Statistical Analysis System Software, Version 9.4; SAS Institute Inc.: Cary, NC, USA, 2012.

19. Howeler, R.H. Cassava mineral nutrition and fertilization. In Cassava, Biology, Production and Utilization; Hillocks, R.J., Thresh, J.M., Bellotti, A.C., Eds.; CABI Publishing: Wallingford, UK, 2002; pp. 115-147.

20. Vanlauwe, B.; Descheemaeker, K.; Giller, K.E.; Huising, J.; Merckx, R.; Nziguheba, G.; Wendt, J.; Zingore, S. Integrated soil fertility management in sub-Saharan Africa: Unravelling local adaptation. Soil 2015, 1, 491-508. [CrossRef]

21. Chen, J.; Lu, G.; Wang, G. Effects of genotype and growing location on iron and zinc contents in sweetpotato (Ipomoea batatas L.). Crop Pasture Sci. 2009, 60, 684-690. [CrossRef]

22. Gopalareddy, K.; Singh, A.M.; Ahlawat, A.K.; Singh, G.P.; Jaiswal, J.P. Genotype-growing locations interaction for grain iron and zinc concentration in recombinant inbred lines of a bread wheat (Triticum aestivum L.) cross. Indian J. Genet. 2015, 75, 307-313.

23. Ande, O.T. Soil suitability evaluation and management for cassava production in the derived Savanna Area of Southwestern Nigeria. Int. J. Soil Sci. 2011, 6, 142. [CrossRef]

24. Wang, Z.H.; Li, S.X.; Malhi, S. Effects of fertilization and other agronomic measures on nutritional quality of crops. J. Sci. Food Agric. 2008, 88, 7-23. [CrossRef]

25. Kirnak, H.; Kaya, C.; Higgs, D.; Gercek, S. A long-term experiment to study the role of mulches in the physiology and macro-nutrition of strawberry grown under water stress. Aust. J. Agric. Res. 2001, 52, 937-943. [CrossRef]

26. Maathuis, F.J. Physiological functions of mineral macronutrients. Curr. Opin. Plant Biol. 2009, 12, 250-258. [CrossRef]

27. Chávez, A.L.; Bedoya, J.M.; Sánchez, T.; Iglesias, C.; Ceballos, H.; Roca, W. Iron, carotene, and ascorbic acid in cassava roots and leaves. Food Nutr. Bull. 2000, 21, 410-413. [CrossRef]

28. Narayanan, N.; Beyene, G.; Chauhan, R.D.; Gaitán-Solís, E.; Gehan, J.; Butts, P.; Siritunga, D.; Okwuonu, I.; Woll, A.; Jiménez-Aguilar, D.M.; et al. Biofortification of field-grown cassava by engineering expression of an iron transporter and ferritin. Nat. Biotechnol. 2019, 37, 144-151. [CrossRef] [PubMed] 
29. Omondi, J.O.; Lazarovitch, N.; Rachmilevitch, S.; Kukew, T.; Yermiyahu, U.; Yasuor, H. Potassium, and storage root development: Focusing on photosynthesis, metabolites, and soluble carbohydrates in cassava. Physiol. Plant. 2020, 169, 169-178. [CrossRef]

30. Burns, A.E.; Gleadow, R.M.; Zacarias, A.M.; Cuambe, C.E.; Miller, R.E.; Cavagnaro, T.R. Variations in the chemical composition of cassava (Manihot esculenta Crantz) leaves and roots as affected by genotypic and environmental variation. J. Agric. Food Chem. 2012, 60, 4946-4956. [CrossRef] [PubMed]

31. Akin-Idowu, P.E.; Asiedu, R.; Maziya-Dixon, B.; Odunola, A.; Uwaifo, A. Effects of two processing methods on some nutrients and anti-nutritional factors in yellow yam (Dioscorea cayenensis). Afr. J. Food Sci. 2009, 3, 086-093.

32. Ismail, F.; Anjum, M.R.; Mamon, A.N.; Kazi, T.G. Trace metal contents of vegetables and fruits of Hyderabad retail market. Pak. J. Nutr. 2011, 10, 365-372. [CrossRef]

33. Gregorio, G.B. Progress in breeding for trace minerals in staple crops. J. Nutr. 2002, 132, 500S-502S. [CrossRef]

Publisher's Note: MDPI stays neutral with regard to jurisdictional claims in published maps and institutional affiliations.

(C) 2020 by the authors. Licensee MDPI, Basel, Switzerland. This article is an open access article distributed under the terms and conditions of the Creative Commons Attribution (CC BY) license (http://creativecommons.org/licenses/by/4.0/). 